\title{
LA REVISTA PERUANA DE MEDICINA EXPERIMENTAL Y SALUD PÚBLICA ASUME CON PROPÓSITOS RENOVADOS SUS RESPONSABILIDADES PARA EL 2019
}

\author{
THE PERUVIAN JOURNAL OF EXPERIMENTAL MEDICINE AND PUBLIC HEALTH ASSUMES \\ ITS RESPONSIBILITIES FOR 2019 WITH RENEWED PURPOSE
}

\author{
Zuño Burstein ${ }^{1}$, J. Jhonnel Alarco²
}

\begin{abstract}
El director de la Revista Peruana de Medicina Experimental y Salud Pública, órgano oficial de difusión científica del Instituto Nacional de Salud (INS) y brazo científico del Ministerio de Salud del Perú, ha expresado en su informe de gestión del 2018 que la calidad científica de la revista ha mantenido sus altos estándares en los últimos años, logrando ser calificada como una de las mejores en el campo de salud pública a nivel latinoamericano y la mejor revista médico-científica peruana.
\end{abstract}

Este logro es posible gracias al invalorable y destacado esfuerzo de su equipo editorial, liderado por su director, el editor general Méd. César Cabezas, y el grupo de editores médicos, laboralmente comprometidos e identificados con su importante responsabilidad, encabezados por el editor científico Franco Romaní y los editores adjuntos, Hugo Arroyo, Jhonnel Alarco y Akram Hernández, con el destacado y eficaz apoyo de la Lic. Bertha Huarez como asistente editorial y responsable secretarial del comité de la revista, así como con la contribución del Méd. Leonardo Rojas como coordinador administrativo, y la asistencia operativa y editorial de la Ing. Leonor Tenorio, Téc. Milagros Orejón y la secretaria Priscilla Silva.

Tal como lo hemos señalado reiteradamente ${ }^{(1-4)}$, nuestra revista esta puesta al servicio de la salud, en especial de nuestro país y de la región latinoamericana. Recibimos las colaboraciones cientificas en las diferentes secciones, no sólo del Perú, sino también de otras partes del mundo. Desde el 2016, año en que se instauró nuestro sistema de gestión OJS (Open Journal System) se ha identificado a 1885 autores, cuya procedencia mayoritaria es de Perú, seguido de Colombia, Ecuador, México, Chile, Venezuela, España, Estados Unidos, Argentina, Cuba y Brasil (5). Respecto a la contribución de científicas mujeres, hemos identificado mayor proporción de autoras en algunos países (Tabla 1).

Nuestro objetivo no sólo se limita al proceso editorial de los artículos que nos remiten, sino también tenemos el propósito, felizmente logrado desde hace varios años, de jugar un rol promotor de la salud pública en Perú ${ }^{(6)}$, mediante la visualización, debate y promoción de la investigación en problemas sanitarios. Para ello, nuestro comité editorial selecciona cuidadosamente un tema relevante para cada número de la revista, el cual es desarrollado con artículos de simposio y en otras secciones de la revista, y además realizamos reuniones conjuntas con la Academia Nacional de Medicina a la salida de cada número para llegar a recomendaciones y conclusiones que son elevadas a los decisores y poderes públicos del Estado.

Tal como se ha señalado en el editorial del número anterior ${ }^{(7)}$, comenzamos este año con el tema: «Formación de Profesionales de la Salud en el Perú» invitando abiertamente y por invitaciones especiales a las instituciones y personalidades académicas universitarias y gubernamentales para que nos envíen sus contribuciones a las diferentes secciones de la Revista y sobre todo a la sección de simposio.

Hemos solicitado colaboraciones sobre los diferentes aspectos de la formación, especialización y capacitación permanente de los profesionales de la salud. Se ha considerado aspectos como la competencia y eficacia de las instituciones dedicadas a la docencia, promoción y estímulo de la investigación médico científica y su difusión informativa; los recursos económicos y financiamiento de la docencia profesional en las universidades públicas y privadas; estrategia y planificación de la formación ética de los profesionales dedicados a la salud en sus diferentes niveles formativos; criterio de calificación, acreditación y licenciamiento de los centros de enseñanza de nivel 
Tabla 1. Procedencia según género de los autores que ingresan su manuscrito al proceso editorial en la Revista Peruana de Medicina Experimental y Salud Pública, 2016-2018

\begin{tabular}{|c|c|c|c|}
\hline \multirow{2}{*}{ País } & Hombre & Mujer & Total \\
\hline & n (\%) & n (\%) & $\mathrm{n}(\%)^{\mathrm{b}}$ \\
\hline Perú & $842(60,0)$ & $561(40,0)$ & $1403(74,4)$ \\
\hline Colombia & $69(45,4)$ & $83(54,6)$ & $152(8,1)$ \\
\hline Ecuador & $49(49,5)$ & $50(50,5)$ & $99(5,3)$ \\
\hline México & $31(60,8)$ & $20(39,2)$ & $51(2,7)$ \\
\hline Chile & $11(32,4)$ & $23(67,6)$ & $34(1,8)$ \\
\hline Venezuela & $15(53,6)$ & $13(46,4)$ & $28(1,5)$ \\
\hline España & $18(78,3)$ & $5(24,7)$ & $23(1,2)$ \\
\hline Estados Unidos & $14(82,4)$ & $3(17,6)$ & $17(0,9)$ \\
\hline Argentina & $8(53,3)$ & $7(46,7)$ & $15(0,8)$ \\
\hline Cuba & $11(78,6)$ & $3(24,4)$ & $14(0,7)$ \\
\hline Brasil & $2(15,4)$ & $11(84,6)$ & $13(0,7)$ \\
\hline Otros países ${ }^{a}$ & $24(66,7)$ & $12(33,3)$ & $36(1,9)$ \\
\hline Total & 1094 & 791 & 1885 \\
\hline
\end{tabular}

universitario y no universitario con la responsabilidad y logros de la Superintendencia Nacional de Educación Superior Universitaria (SUNEDU); entre otros.

Se ha recibido colaboraciones que han sido sometidas al proceso de revisión por pares y que luego de su publicación en este número serán incluidos en el temario de la Conferencia Conjunta con la Academia Nacional de Medicina, que se realizará el día 25 de abril en el Colegio Médico del Perú.

Entre las contribuciones relacionados al tema podemos mencionar los siguientes artículos de simposio: «Ética y bioética en el pregrado de medicina: una propuesta» de autoría del Dr. Jaime Millás-Mur; «La especialidad de Gestión en Salud en la Universidad Nacional Mayor de San Marcos: 30 años formando gestores para el sector salud» de autoría del Dr. Germán Felipe Rivera Del Río; «Producción científica y licenciamiento de escuelas de medicina en el Perú» de autoría del Dr. Percy Mayta-Tristán; y «Formación ética como soporte del desarrollo moral del alumno de carreras de Ciencias de la Salud: hacia una enseñanza centrada en las necesidades del estudiante» cuyo autor es el reconocido académico Dr. Alberto Perales.
Además, nuestro comité ha considerado pertinente la publicación del artículo original breve titulado: «Número de nefrólogos, servicios de hemodiálisis y tendencia de la prevalencia de enfermedad renal crónica en el Ministerio de Salud de Perú» de autoría del Dr. Percy Herrera-Añazco y una carta científica titulada: «Variación del puntaje obtenido en el examen nacional de obstetricia según licenciamiento universitario, 2018» de autoría de obstetra Víctor Hugo Moquillaza-Alcántara.

Asimismo, contamos con la valiosa contribución de la Dra. Graciela Risco de Dominguez decana de la Universidad Tecnológica del Perú, quien nos presenta los indicadores de calidad más importantes a tomarse en cuenta en el próximo licenciamiento de los programas de pregrado de Medicina, a través de su editorial titulada: «Nuevos factores que inciden sobre la calidad de la educación médica y de las profesiones de salud».

También queremos resaltar en este número la figura de la destacada profesora doctora Sonia Burstein Alva, quién un año antes de su sensible fallecimiento a los 84 años de vida (16 de octubre del 2018), y años después de haberse retirado de la actividad docente y de su práctica profesional privada, logró escribir un libro con lo que consideraba una contribución de su experiencia personal a los estudiantes y médicos dedicados a su especialidad y a la medicina en general. Esta obra, que no llegó a ser publicada, titulada «Memorias y Experiencias en Microbiología Clínica», contiene algunos capítulos que tienen el carácter de un testimonio de vida, que ha sido publicado en el Boletín $\mathrm{N}^{\circ} 1$ (2019) del INS por ser de interés para el tema de la formación de profesionales de la salud en Perú. Próximamente, el texto completo de la obra será publicado, por sus contribuciones tecnológicas y conceptuales a los diferentes procedimientos de laboratorio y diagnóstico microbiológico.

Por último, alentamos a la comunidad científica a enviar sus contribuciones para el segundo número de nuestra revista, cuyo tema central será la «Atención médica en el Perú». También instamos a los autores e instituciones de investigación a enviar sus contribuciones, ajustándose a los requisitos establecidos en las normas para autores y seguir las pautas vinculadas con la ética en publicación científica.

\section{REFERENCIAS BIBLIOGRÁFICAS}

1. Burstein Z. Presentación pública de la versión actual y reseña histórica de la Revista Peruana de Medicina Experimental y Salud Pública. Rev Peru Med Exp y Salud Publica. 2011;28(1):8-12.

2. Cabezas C. Necesidad de la Investigación en Salud para contribuir a la equidad, la salud y el desarrollo. Rev Peru Med Exp Salud Pública. 2010;27(3):310-11.

3. Burstein Z, Cabezas C. La Revista Peruana de Medicina Experimental y Salud Pública continúa al servicio de la salud pública del Perú y Latinoamérica. Rev Peru Med Exp Salud Pública. 2016; 33(1):10-2.

4. Burstein Z, Romaní F. La Revista Peruana de Medicina Experimental y Salud Pública en el contexto político nacional. Rev Peru Med Exp Salud Pública. 2018; 35(1):5-6.

5. Burstein Z. Apreciación crítica a la gestión 2016 y perspectivas al nuevo año $2017 \mathrm{de}$ la Revista Peruana de Medicina Experimental y Salud Pública. Rev Peru Med
Exp Salud Pública. 2017;34(1):7-10. doi 10.17843/rpmesp.2017.341.2761.

6. Soto A, Burstein Z. Salud pública e investigación: la agenda pendiente. Rev Peru Med Exp Salud Publica. 2016;33(3):399-400. doi:10.17843/rpmesp.2016.333.2317.

7. Solari L, Burstein Z. La Revista Peruanade Medicina Experimental y Salud Pública al término del 2018 y su responsabilidad para el próximo año. Rev Peru Med Exp Salud Publica. 2018;35(4): 561-2. doi:10.17843/rpmesp.2018.354.4113. 